\title{
Erratum to: Effect of Different Gentamicin Dose on the Plasticity of the Ribbon Synapses in Cochlear Inner Hair Cells of C57BL/6J Mice
}

\author{
Liping Chen $\cdot$ Siqing Xiong $\cdot$ Yi Liu $\cdot$ Xiuli Shang
}

Published online: 4 October 2012

(C) Springer Science+Business Media New York 2012

Erratum to: Mol Neurobiol

DOI 10.1007/s12035-012-8312-7

We would like to clarify that there are some changes to the identity of the first author. She first was a student of the Department of Neurology, The First Affiliated Hospital of China Medical University, and she works in the Department of Neurology, The Ji'an Central People's Hospital.

The online version of the original article can be found at http:// dx.doi.org/10.1007/s12035-012-8312-7.

L. Chen $\cdot X$. Shang $(\bowtie)$

Department of Neurology, The First Affiliated Hospital of China

Medical University,

92 North Second Road,

Shenyang 110001, China

e-mail: chenzijoycexiong@126.com

L. Chen · Y. Liu

Department of Neurology, The Ji'an Central People's Hospital,

106 Jing Gang Shan Road,

Ji' an City Jiangxi Province 343000, China

\section{S. Xiong}

Department of Urinary Surgery,

The Ji'an Central People's Hospital,

106 Jing Gang Shan Road,

Ji' an City Jiangxi Province 343000, China 